\title{
Lidil
}

Revue de linguistique et de didactique des langues

$31 \mid 2005$

Corpus oraux et diversité des approches

\section{Ce que l'oral nous a appris sur la syntaxe du français}

Paul Cappeau

\section{OpenEdition}

Journals

Édition électronique

URL : http://journals.openedition.org/lidil/179

DOI : $10.4000 /$ lidil. 179

ISSN : $1960-6052$

Éditeur

UGA Éditions/Université Grenoble Alpes

Édition imprimée

Date de publication : 1 juin 2005

Pagination : 157-177

ISBN : 2-914176-12-0

ISSN : $1146-6480$

Référence électronique

Paul Cappeau, "Ce que l'oral nous a appris sur la syntaxe du français », Lidil [En ligne], 31 | 2005, mis en ligne le 05 octobre 2007, consulté le 21 avril 2019. URL : http://journals.openedition.org/lidil/179 ; DOI : 10.4000/lidil.179

Ce document a été généré automatiquement le 21 avril 2019

(C) Lidil 


\title{
Ce que l'oral nous a appris sur la syntaxe $\mathrm{du}$ français
}

\author{
Paul Cappeau
}

1 Le thème de ce numéro offre un bon prétexte pour dresser, sur quelques points, un bilan de l'intérêt de l'oral pour une meilleure connaissance de la syntaxe du français. Travailler à partir de données orales impose un certain nombre de contraintes, qu'il est peut-être utile de rappeler (en partie du moins). Il est, par exemple, indispensable de disposer de corpus fiables: ce qui induit que leur constitution a été préparée (il ne suffit pas de collecter des données au hasard pour constituer un corpus exploitable de façon satisfaisante) et qu'ils ont été vérifiés avec minutie. L'exploitation des corpus doit ensuite tenir compte de certaines tendances régulières dans l'organisation et la distribution des faits syntaxiques. Notre première partie précisera les différentes phases qui permettent de constituer des corpus oraux. La deuxième partie fournira quelques exemples de savoirs nouveaux que l'oral nous a permis de faire ressortir.

Travailler sur corpus

2 De nos jours, la plupart des linguistes semblent accorder de l'intérêt aux exemples réels, si l'on en juge par les énoncés cités dans les articles, dont beaucoup sont référencés pour l'écrit (tirés par exemple de la presse ou de Frantext). Le même mouvement touche les énoncés oraux qui sont assez fréquemment utilisés (il suffit, là encore, de consulter des articles récents). Les exemples de cette dernière catégorie peuvent provenir de deux sources : soit ils sont récupérés à la volée, soit ils sont tirés de corpus existants. Prélever des exemples dans son environnement offre l'avantage de facilité de mise en œuvre : une certaine vigilance pour identifier les énoncés intéressants, un bout de papier et un stylo suffisent généralement. Mais ce butinage présente de multiples travers qui en limitent l'intérêt : la fiabilité des exemples notés est sujette à caution (du moins s'ils n'ont pas été enregistrés), car tout transcripteur sait à quel point les phénomènes de reconstruction doivent être combattus (si l'on s'intéresse à la production). Dans une situation d'échange réel, nous ne percevons que rarement les reformulations que nous effectuons ou qu'opère notre interlocuteur. On peut illustrer ce risque à travers l'exemple suivant :

- il y a il y a tout un tas de de il existe tout un tas de recettes miracles qui dont les plus courantes sont effectivement le dentifrice (CA) 
$$
\text { interviennent, comme dans le passage de il y a a il existe, ou de qui à dont, et de recuper }
$$
interviennent, comme dans le passage de il y a à il existe, ou de qui à dont, et de récupérer ces indices qui signalent l'élaboration de l'énoncé. En situation d'interaction, l'auditeur perçoit le sens global de l'énoncé, une séquence dépouillée des phénomènes de reformulation et serait, vraisemblablement, enclin à restituer l'énoncé suivant :

- il existe tout un tas de recettes miracles dont les plus courantes sont effectivement le dentifrice

On peut craindre aussi que le contexte linguistique noté soit assez réduit (les limites de la mémoire à court terme autorisent un maximum de 15 mots!) et peu contrôlé (le transcripteur relèvera-t-il toutes les informations utiles ?). On se prive, de fait, de la possibilité d'élargir les paramètres d'analyse. L'exemple se présente finalement sous une forme figée : il est exclu à tout jamais d'en étendre le contexte.

5 De plus, ces exemples ne permettent pas une approche quantifiée. Comment, en effet, mesurer l'importance ou la rareté des faits relevés? On peut penser que le chercheur sera vigilant pour relever les tournures qu'il recherche et moins attentif pour d'autres tours. Il devient, dès lors, impossible de s'appuyer sur ces exemples pour fournir des informations fiables sur la représentativité des données. Bref, travailler avec des exemples isolés fait courir le risque de disposer d'un matériau hétéroclite. La description que l'on en tire comporte une marge d'incertitude qui laisse insatisfait'.

6 L'appui sur corpus ${ }^{2}$ présente de nombreux intérêts (Pusch \& Raible, 2002) ce qui explique pourquoi, malgré le très lourd travail que représentent le choix des enregistrements à effectuer, la transcription et l'édition de corpus oraux, il n'est pas envisageable de se passer de telles données. Il est peut-être utile de détailler ces différents points.

Le choix des enregistrements

7 Le chercheur qui souhaite décrire la langue en exploitant des corpus se trouve, par làmême, très dépendant de la qualité des données sur lesquelles il s'appuie. En effet, généralement, il considère que le corpus utilisé lui fournit une image fidèle de ce qu'est la langue. Pour cela, il extrapole à partir du corpus dont il dispose. Cette démarche a subi de nombreuses attaques (Meyer, 2002), émanant notamment de théoriciens convaincus qu'on ne pouvait trouver la totalité des faits langagiers à partir de données recueillies et qu'il valait donc mieux tirer parti de sa connaissance de la langue pour proposer l'analyse de séquences produites à la demande. Le recours à de grands corpus informatisés qui permettent d'accéder à des ressources abondantes réduit fortement ces critiques. Certes, il serait naîf de penser qu'un corpus contiendra la totalité des possibles mais, au moins, on peut espérer qu'il fournisse les grandes tendances des principaux faits de langue. Pour autant, il ne faut pas considérer comme négligeable la faiblesse potentielle de la description. Pour que l'appui sur un corpus puisse apporter quelques lumières sur ce qu'est la langue, il faut veiller à ce qu'il présente à la fois une taille suffisante (ce qui laisse espérer qu'il contienne des faits langagiers représentatifs) et une certaine diversité (qui permet de rencontrer des faits qui ne sont produits que dans certaines situations). Ces deux facteurs pallient les deux écueils que Benoît Habert (2000) rappelle : l'incertitude (quand le corpus est trop petit ${ }^{3}$ ) et la déformation (quand il est trop uniforme).

8 Durant une première période, les corpus du GARS ont été collectés de façon quasi-libre (Blanche-Benveniste, 2000). Le but était de disposer de nombreux enregistrements de langue orale réelle à une époque où celle-ci restait peu étudiée. Pour éviter de conforter les clichés liés à l'oral ${ }^{4}$ (le caractère familier des échanges, l'aspect chaotique et 
déstructuré de la parole, etc.), les conversations ont été peu retenues comme source des enregistrements. Cette première période a permis d'obtenir un corpus (dénommé CorpAix) possédant une taille «critique » de un million de mots, qui a servi d'appui à de nombreuses analyses en morphosyntaxes. Il apparaît cependant nécessaire de s'interroger, d'une part sur les caractéristiques et les limites de ce corpus et d'autre part, sur les façons de l'enrichir et de l'améliorer. Ainsi, les récits de vie, nombreux dans CorpAix, présentent une situation particulière qui fait certainement surgir certains faits linguistiques mais n'est pas propice à la réalisation d'autres. De même, les situations vécues sont rarement présentes : il manque des interactions comme des échanges dans le cadre familial, des enregistrements en situation de travail (interactions dans un commerce, personnes en train d'exercer leur profession, etc.). Le corpus est probablement déséquilibré au profit des situations commentées (ce que la forme de l'interview très fréquemment utilisée a vraisemblablement favorisé). On se prive notamment de séquences locatives avec contre (contre le mur, etc.) ou d'énoncés exclamatifs (tels que oh comme il est joli!) qui sont, de fait, quasi absents de CorpAix.

Il devient alors délicat d'assimiler ce corpus au français parlé, et en particulier de considérer que décrire un point de langue dans CorpAix fournit la distribution effective de celui-ci dans la langue orale. C'est par un raccourci pratique que l'on peut parfois adopter cette formulation mais il conviendrait d'être plus nuancé. Ce corpus a permis de développer des analyses originales car il contenait de l'oral véritable et permettait de travailler sur des données non retenues jusque là. Certaines des limites de ce corpus commencent à ressortir et montrent la nécessité de l'enrichir. La constitution d'un corpus français de référence' (identifié par FR ou CRFP pour Corpus de référence de français parlé) permet d'effectuer des comparaisons et de vérifier que des écarts sensibles (qui tiennent à la composition des données collectées) apparaissent entre ces deux recueils de données orales. Cappeau (2004) illustre ces différences dans la distribution des pronoms toniques en position sujet. La part de langue formelle est un peu plus élevée dans FR, ce qui modifie la part que représentent certaines tournures. Ainsi, les exemples dans lesquels nous est encadré par c'est... qui sont plus nombreux :

- c'est nous qui organisons tout en fait (FR)

10 L'augmentation de la taille de CorpAix passe maintenant moins par un accroissement aveugle et important des données déjà rassemblées que par une recherche de diversification encore plus importante des situations recueillies. En effet, doubler la taille d'un corpus n'est pas nécessairement intéressant si les nouvelles données diffèrent trop peu des anciennes. Ont par exemple été ajoutées des conversations, ainsi que des données obtenues en situations formelles (visites guidées, médiations judiciaires dans les médias, etc.), pour étendre la diversité. La variété est actuellement envisagée au travers du filtre des genres et repose sur l'hypothèse (vérifiée pour partie) que divers phénomènes morpho-syntaxiques sont sensibles à des paramètres externes et se rencontrent donc plus dans certaines situations, dans certains " genres ${ }^{8} »$ (Biber, 1988 ; Malrieu \& Rastier, 2001). Dès lors, accroitre la variété des situations, c'est se donner les moyens de repérer plus aisément certains faits de langue.

La transcription

11 La transcription n'est pas un simple travail de dactylographie. Cette position maintes fois défendue (Blanche-Benveniste \& Jeanjean, 1987) doit être rappelée car elle explique, pour une part, la difficulté à obtenir des données orales en grande quantité. C'est une activité qui exige une véritable formation. 
12 La transcription comporte plusieurs phases qui rendent ce travail très lourd: une première écoute permet de se sensibiliser à la voix, puis le travail de transcription proprement dit demande, pour certains passages, de multiples écoutes avant de fournir une version acceptable. En effet, il ne s'agit pas simplement de noter ce que l'on entend, il faut encore donner à la séquence sonore une forme exploitable syntaxiquement. Cette exigence n'implique pas de reconstruire le discours mais plutôt de rechercher la forme la plus adéquate quand plusieurs solutions sont envisageables. Ainsi, pour transcrire la séquence sonore suivante/lykivøsa/, on peut retenir au moins deux solutions :

(a) Luc y veut ça

(b) Luc il veut ça

13 La version (a) est proche de certaines représentations de la langue parlée que la littérature ou la presse nous donnent parfois, comme cet exemple récent Vous pouvez me croire que nous, $y$ viennent pas nous asticoter... trouvé dans Libération (29 mars 2004). On est là dans le cliché. La forme écrite $y$ joue un double rôle : elle est censée reproduire au mieux la forme sonore /i/ et elle donne une impression de français plus relâché par écart de la forme attendue ils'. Le premier argument n'est pas vraiment satisfaisant puisque l'on fait semblant d'ignorer que le pronom ils (ou il) peut selon les contextes être prononcé avec ou sans /1/. La graphie $y$ n'est donc pas un bon choix si l'on souhaite mimer la prononciation. De plus, seule la version (b) correspond à un découpage syntaxique plausible, c'est pourquoi elle sera préférée. De même, dans la paire qui suit, on écartera la version (a) et on ne retiendra que la version (b) :

(a) i-z-ont pas réussi

(b) ils ont pas réussi

Certaines séquences sonores sont particulièrement délicates car les locuteurs adoptent des prononciations qualifiées de "rapides » (ou relâchées) qui exigent une vigilance plus grande du transcripteur. Divers contextes rendent difficile la perception de certaines formes grammaticales. Lorsque la même voyelle se répète, il est parfois difficile d'identifier les deux occurrences, les transcripteurs peuvent être tentés de n'en retenir qu'une. D'où des difficultés pour différencier, dans certains usages, l'imparfait et le passé composé :

- avec ce qu'on a appelé

- avec ce qu'on appelait

15 Un transcripteur confirmé pourra parfois distinguer les deux emplois. Dans certains cas, il devra maintenir les deux solutions sans pouvoir trancher :

- ce qu'on / a appelé, appelait /

D'autres points doivent faire l'objet d'une attention particulière lors de la transcription. Ainsi, dans l'énoncé des entreprises qui ont été rachetées, la suite qui ont peut être réalisée avec ou sans la présence du /i/. Dans ce dernier cas, on n'écrira toutefois pas qu'ont car cette suite serait non interprétable du point de vue morphosyntaxique.

De façon plus générale, on considère qu'une transcription n'est pas un produit totalement "objectif», mais qu'elle dépend étroitement de l'usage qui sera fait du corpus. Les approches communicationnelles sont ainsi sensibles à des paramètres tels que la longueur des silences, certaines caractéristiques de la voix, etc. qui sont en général ignorées dans les transcriptions établies pour développer des analyses syntaxiques. C'est pourquoi, d'ailleurs, les conventions varient selon les buts que l'on assigne aux études qui doivent être conduites à partir des transcriptions. Des équipes différentes ne transcriront 
pas de façon semblable un même enregistrement. Les transcriptions élaborées par le GARS $^{10}$ et actuellement utilisées par DELIC sont soumises à diverses exigences : le respect des données (qui conduit à ne pas ajouter ni enlever de séquences non perçues), la prise en compte de quelques faits de prononciation et la préférence accordée aux séquences qui peuvent recevoir une interprétation syntaxique quand plusieurs solutions se présentent.

C'est en s'appuyant sur ces principes que l'on choisit de toujours transcrire il y a les séquences qui peuvent être prononcées /ja/, /ija/, /ilja/ ou /ilija/. Les diverses réalisations sonores envisageables sont traitées comme des variantes qui renvoient toutes à la même séquence syntaxique : il y $a$. Il a paru préférable de conserver le clitique il qui est conforme à la structure habituelle du français.

La volonté de fournir en priorité une séquence qui peut recevoir une analyse syntaxique aboutit parfois à des solutions qui peuvent perturber le néophyte ou le sociolinguiste qui tient légitimement à noter les variations de prononciation. Ainsi, dans l'exemple cidessous (dans lequel les signes + notent des pauses) :

et là on est allé voir les + les vrais camps euh + avec les vrais Sénégalais euh + le truc typique quoi + où c'est qui tuaient la poule devant nous là pour la manger à midi (FR - version non corrigée)

il est impossible de conserver la suite où c'est qui tuaient (véritable monstre syntaxique !) qu'il faut comprendre comme où c'est qu'ils tuaient. L'erreur provient de la séquence sonore /ki/ qui a été réanalysée en tenant compte des habitudes de prononciation du pronom sujet $i l s$ devant consonne.

21 La transcription, on l'a dit, doit se conformer à une charte constituée par les conventions adoptées. Le GARS a adopté des conventions assez «dépouillées » (le texte est, pour l'essentiel, donné en orthographe standard, les indications sur la situation sont très réduites, les particularités de prononciation sont limitées, etc.) en considérant que l'analyse syntaxique pouvait, pour partie, se passer de ces divers paramètres. Ce qui était vrai pour les études de morphosyntaxe l'est moins pour l'approche macrosyntaxique. Ce nouveau besoin n'a toutefois pas conduit à modifier des conventions qui assurent une grande lisibilité aux transcriptions". La technologie vient, de fait, pallier la pauvreté des informations supra-segmentales que le texte écrit comporte : les transcriptions alignées (dans lesquelles le texte est solidaire de la version sonore) permettent à la fois de travailler sur la version brute du texte et de l'enrichir avec la version sonore.

L'édition des corpus

Le travail de vérification des transcriptions est le versant ingrat, souvent méconnu (et pourtant ô combien indispensable!) de l'édition des corpus. Il est essentiel que les transcriptions soient validées par un « expert " pour pouvoir être ensuite exploitées. La transcription de départ contient toujours des erreurs d'écoute et d'orthographe qui peuvent gêner l'exploitation des données. Les corrections portent notamment sur des problèmes d'orthographe (a), de méconnaissance culturelle (b) ou d'écoute liés à l'interprétation syntaxique (c) :

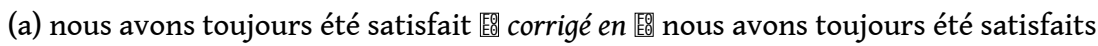

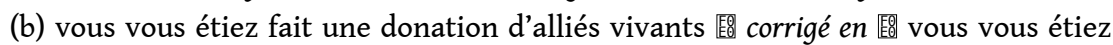

fait une donation au dernier vivant

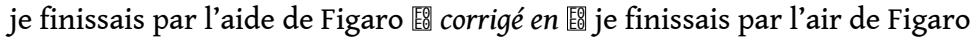

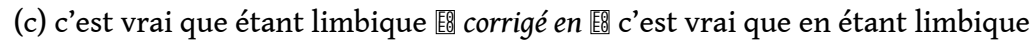

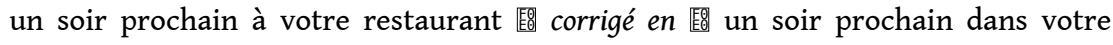

restaurant

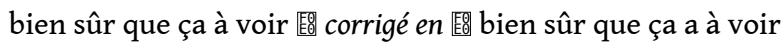


Pour donner une idée de l'importance de cette partie du travail, pour un corpus de 4000 mots, un correcteur peut aller jusqu'à 160 modifications de la transcription originale ! $\mathrm{Ce}$ travail lourd est la garantie que les transcriptions seront de qualité et permettront de bâtir des analyses fondées. Par expérience, on sait pourtant que le travail de transcription n'est jamais totalement achevé. Sur des gros corpus, toute nouvelle lecture permet de détecter quelques « dernières » erreurs...

L'apport de l'oral à la description syntaxique

Il n'est pas envisageable de dresser un inventaire (même succinct) des connaissances que l'oral a apporté dans les différentes rubriques d'une grammaire. Plusieurs raisons rendent ce travail impossible ${ }^{12}$ d'une part, un relevé exhaustif n'a pas été établi jusqu'ici ${ }^{13}$ ce qui nécessiterait donc un lourd travail; d'autre part, et c'est probablement l'obstacle principal, une telle entreprise pourrait laisser supposer qu'il existe (du point de vue syntaxique) une réelle coupure entre oral et écrit, position qui a été rejetée, dès l'origine, par les membres du GARS.

Que proposer alors pour répondre tout de même à la question que pose le titre de cet article ? Il a paru plus pertinent d'expliciter ce que le travail avec des corpus oraux nous a permis de comprendre sur l'organisation de la langue ${ }^{14}$. Certaines régularités se dégagent lorsque l'on recherche une forme à l'aide d'un concordancier ${ }^{15}$. Sur un certain nombre de points de langue, les corpus constituent un outil indispensable, notamment lorsqu'il s'agit de connaître la distribution de divers morphèmes ou de structures syntaxiques sur lesquels les locuteurs ont peu d'intuition. Ainsi que sait-on véritablement sur les emplois, du clitique $y$ (Sabio, 2004), de contre (Bilger \& Cappeau, 2003), de une fois (BlancheBenveniste, 1998)? Les corpus oraux sont de précieuses sources d'information dans lesquelles les écarts sont plus tranchés entre formes de grande et de moyenne fréquences ${ }^{16}$. L'approche sur corpus oraux permet d'avoir une approche hiérarchisée des phénomènes qui tient compte de l'importance quantitative des phénomènes traités. Ainsi, Frédéric Sabio (2004) est conduit à distinguer trois types d'emplois du pronom y:

- en tant que complément locatif obligatoire d'un verbe (comme dans j'y vais, j'y suis né, j'y suis, etc.). Cet emploi est fréquent quelles que soient les données utilisées ;

- en tant que complément locatif facultatif d'un verbe c'est-à-dire comme complément qui appartient à la valence et peut être réalisé zéro. Dans ce cas, il ne semble pas y avoir de réelle différence de sens que le complément soit ou non réalisé comme dans j'y reste deux semaines vs je reste deux semaines, etc. La présence du complément y est perçue comme la marque d'une langue plus cérémonieuse ;

- en tant que complément non valenciel d'un verbe soit comme un locatif qui peut s'ajouter à n'importe quel verbe. Ce dernier emploi semble réservé à une langue très formelle, quelque peu académique (je connaissais l'Espagne hein + j'y avais des amis)

- De façon générale, l'oral (du moins dans sa composante non formelle que l'on pourra qualifier de tout venant) présente souvent trois particularités bien utiles lorsque l'on développe des descriptions syntaxiques : d'une part, l'inventaire des formes est plus étoffé (en particulier on trouve, dans l'oral non formel, des suites écartées de l'écrit, rejetées par la norme) ; d'autre part, les éléments résiduels ou marginaux (très liés à une préciosité de style) y sont plus rares ; enfin, certaines associations privilégiées peuvent occuper une part importante de la distribution. L'oral permet de dégager plus aisément les axes majeurs de la distribution. Pour illustrer ces différents aspects, nous prendrons quatre exemples : les emplois de contre, le déterminant quelques, les indéfinis en relation avec la position sujet, les participes (passés et présents). 
La distribution de contre

Lorsque l'on recherche les contextes dans lesquels apparaît l'élément contre, on constate (Bilger \& Cappeau, 2003) une forte disparité entre :

- l'oral spontané où par contre représente $52 \%$ des emplois ;

- l'oral formel des hommes politiques où par contre n'occupe plus que $6 \%$ des cas ;

- l'écrit journalistique dans lequel par contre représente $1 \%$ des exemples.

Il n'est, sur ce point, pas possible de tracer une ligne de partage entre productions orales et écrites. Ces résultats montrent que la coupure porte plutôt sur la distinction entre langue formelle et langue tout venant, ce qui appuie la nécessité de disposer de corpus variés pour ne pas s'enfermer dans une représentation étroite et caricaturale de ce que serait l'oral limité à du familier (sur ce point voir le développement en infra). On constate à quel point la norme (qui condamne par contre) intervient dans les productions formelles (tant écrites qu'orales) et pèse sur les distributions observées. Dans l'oral tout venant, contre se réalise majoritairement dans un contexte ignoré par les autres sortes de productions $^{17}$.

Une autre ligne de fracture concerne la nature de la tête dont contre + SN est le complément et peut être reliée à l'importance des catégories verbales et nominales dans les productions (Halliday, 1985). Dans l'oral non formel, les séquences en contre qui introduisent des compléments du verbe sont les plus nombreuses (près de $75 \%$ des exemples) :

- j'ai rien contre elle elle est très bien (FR)

29 Dans la langue écrite, cette proportion n'est plus que de $50 \%$. Dans l'oral formel l'emploi adverbial de contre (i.e. sans réalisation du SN qui suit) est bien représenté, et cela tient à la forte proportion de séquences qui appartiennent à l'oral des politiques comme voter contre:

- ceux qui ont décidé de voter contre pour s'opposer au gouvernement (HP)

Enfin dans la langue écrite, dans la moitié des emplois contre introduit le complément d'une tête nominale :

- quand il a décidé d'engager les hostilités contre Saddam Hussein (Le Monde,

2-10-04)

Ces faits illustrent l'indispensable prise en compte de l'oral pour mieux appréhender la diversité des usages syntaxiques, et soulignent à la fois l'influence des types de productions utilisés sur les résultats obtenus et l'importance de certains faits de lexicalisation sur lesquels les exemples suivants permettront de revenir.

Les emplois de quelques

32 Le déterminant qui apparaît le plus souvent devant le terme instants est quelques. En prenant appui sur l'écrit ${ }^{18}$, au moins quatorze contextes différents ont été rencontrés, dont beaucoup n'existent qu'à un seul exemplaire (ses instants, vos instants, trois instants, etc.) A l'oral, ce terme est plutôt rare et se rencontre dans deux contextes seulement : quelques instants et tous les instants. La distribution est certes épurée mais ne fait pas manquer le contexte le plus caractéristique (alors qu'à l'inverse, on a vu avec contre que l'écrit écartait le contexte le plus répandu à l'oral).

L'exploitation de vaste corpus oraux conduit à s'intéresser aux associations privilégiées ou des séquences préformées (Moreau, 1986) lorsque l'on travaille sur des items grammaticaux. Ainsi, le déterminant quelques se rencontre peu avec des noms qui 
possèdent le trait humain ( $7 \%$ des emplois). Parmi les noms qui ont le trait non humain, une majorité se dégage en faveur des noms temporels (quelques années, quelques jours, quelques mois, quelques instants...). Cette distribution originale incite à se demander si, dans notre activité de production, notre liberté de construction est entière. De tels faits laissent plutôt envisager que nous disposons, à côté des unités de la langue (que nous assemblons de façon relativement libre), de blocs plus importants (prêts à l'emploi dans des structures plus vastes).

Les pronoms indéfinis sujets

L'oral permet aussi parfois de disposer de formes plus nombreuses, qui permettent de mieux comprendre l'organisation du système. Ainsi, pour l'étude des indéfinis, l'apport de l'oral est décisif. Un relevé sur les pronoms indéfinis en position sujet fournit des résultats inattendus (Cappeau \& Deulofeu, à paraître). Le nombre d'exemples rencontrés est très réduit :

- quelques-uns ne parlaient pas anglais du tout (FR)

Le tableau ci-après indique (pour CorpAix) les exemples dans lesquels ces pronoms indéfinis sont directement sujets (comme dans l'exemple qui précède) :

\begin{tabular}{|l||l|l|l|l|}
\hline certains & beaucoup & d'autres & plusieurs & quelques-uns \\
\hline \hline 28 & 10 & 8 & 2 & 2 \\
\hline
\end{tabular}

Tableau 1 - Indéfinis directement sujets.

On pourrait penser a priori que, à l'oral, plutôt que d'employer la forme «simple » du pronom, on privilégie une tournure plus longue telle que il y en a... qui... :

- alors il y en a quelques-uns qui savent (FR)

Le tableau suivant montre que cette piste n'est pas totalement convaincante :

\begin{tabular}{|l|l|l|l|l|}
\hline certains & beaucoup & d'autres & plusieurs & quelques-uns \\
\hline \hline 6 & 19 & 20 & 4 & 0 \\
\hline
\end{tabular}

Tableau 2 - Indéfinis réalisés dans la tournure il y en a... qui.

Comment interpréter les résultats de ce tableau ? Pour des pronoms tels que beaucoup ou d'autres, on observe que la forme longue permet d'augmenter de façon sensible les rares emplois de la forme courte. On se trouverait donc dans un cas de supplétisme grammatical. Mais deux facteurs résistent à cette présentation : pour certains pronoms ( quelques-uns, certains) la forme longue n'apporte aucune augmentation du nombre d'occurrences, globalement le nombre d'exemples cumulés des deux tableaux reste étonnamment faible et conduit à se demander où sont passés les sujets indéfinis non lexicaux. La réponse ne peut être trouvée que lorsque l'on s'écarte de l'écrit. Il faut renoncer à vouloir présenter les phénomènes en terme de défaillance (considérer par exemple que l'oral serait moins apte à recourir à une forme synthétique - i.e. un pronom indéfini en position sujet - et privilégierait des formes renforcées comme il y en a beaucoup qui, etc.) mais plutôt considérer chacune des prédications (la construction 
directe et celle en il y en a... qui...) avec ses spécificités. On peut alors envisager une vision plus complexe des indéfinis en relation avec la position sujet :

a) Dans la prédication directe, le sujet certains occupe une place à part. Il est d'emploi bien plus fréquent que les autres pronoms :

- mais certains n'étaient pas compétnets ils étaient vieux (CA)

L'emploi de certains est très sensible au type de production utilisé. Ainsi, dans les corpus formels d'interviews politiques, on rencontre de nombreuses occurrences de certains avec des verbes de parole :

- certains disent même que vous avez relancé la lutte (HP)

b) La prédication il y en a... qui se rencontre principalement avec deux valeurs presque opposées :

- en relation avec une quantification vague, non précisée par un pronom. Il s'agit de la séquence il y en a qui :

- il y en a qui sont plus ou moins adaptés à certains sols (CA)

Dans ce cas, il est exclu d'établir une correspondance avec la construction directe, puisque le sujet doit être exprimé en français contemporain :

- * $\varnothing$ sont plus ou moins adaptés à certains sols

- pour exprimer une quantité égale à un (il y en a un qui) :

- il y en a un qui part dans une demi-heure c'est le dernier (CA)

Il n'y a, à nouveau, pas de réelle concurrence avec la prédication directe qui admet mal le sujet un ${ }^{19}$ :

- ?? un est parti il y a une demi-heure

Les autres emplois (en relation avec des pronoms indéfinis) sont plutôt minoritaires, et apparaissent presque marginaux. Ainsi, une présentation des indéfinis en relation avec la position sujet qui tient compte de l'oral doit prévoir de multiples subdivisions :

Tableau 3 - Les indéfinis en relation avec le sujet.

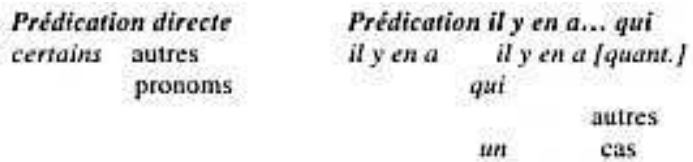

L'utilisation des participes passés et présents

47 Une dernière illustration permettra de préciser certains des aspects déjà dégagés. Elle concerne les participes passés (Blanche-Benveniste, 1998) et les participes présents (Bilger \& Cappeau, 2004) employés sans auxiliaires. Les distributions de ces deux catégories présentent de grandes similitudes. Trois grands cas peuvent être identifiés :

48 a) Certains usages sont limités à une langue marquée comme soutenue ou littéraire. Ils peuvent être identifiés comme des marqueurs de langue formelle. C'est le cas des emplois que l'on pourrait qualifier de "directs ». Pour le participe passé, Blanche-Benveniste (1998) signale que l'usage littéraire autorise, dans ce cas, une grande variété de lexèmes verbaux :

- Rentré chez eux, le marmot fut gâté comme un prince. (Flaubert) 
articipe présent réalisé directement est assez rare à l'oral, et semble constituer un marqueur de genre (il est par exemple assez fréquent dans l'oral professionnel des guides $\left.{ }^{20}\right)$ :

- toutes les couches archéologiques correspondant à la date euh à la à l'époque de la façade avaient été bouleversées euh au dix-septième siècle (CA)

b) Un petit nombre d'unités lexicales échappent à la contrainte précédente et se rencontrent donc plus facilement dans le français tout venant. Ainsi, pour le participe passé, on trouve de nombreux exemples avec arrivé, sorti ou fini :

- il est blanc + parce que arrivé au nord + il n'a plus la couleur (FR)

Pour les participes présents, c'est principalement la forme étant qui est utilisée sans trop de contraintes :

- bon étant assez + habile de mes mains + je pouvais réaliser des petits dessins

+ sympathiques (FR)

c) Il existe des contextes spécifiques qui permettent d'étendre l'emploi des participes sans auxiliaire à tous les lexèmes verbaux. C'est la tournure une fois qui remplit ce rôle pour le participe passé :

- une fois choisie + l'équipe elle était faite pour la saison (FR)

Pour le participe présent, la forme précédée de en (le gérondif) autorise l'emploi de verbes variés. La séquence en + participe présent se rencontre dans des corpus divers et ne semble plus devoir être rattachée à un genre :

- et en tournant à gauche + on se si- on se dirige + vers la rue de la République (FR) L'essentiel de la distribution de ces deux formes verbales peut donc être synthétisée dans le tableau suivant :

Tableau 4 - Ossature de la distribution des participes.

\begin{tabular}{|c|c|c|c|}
\hline & \multirow{2}{*}{$\begin{array}{l}\text { langue } \\
\text { formelle }\end{array}$} & \multicolumn{2}{|c|}{ oral tout venlant } \\
\hline & & forme seule & forme etoffée \\
\hline & tous les verbes & $\begin{array}{l}\text { Jexique } \\
\text { particulier }\end{array}$ & tous les verbes \\
\hline $\begin{array}{l}\text { participe } \\
\text { passe. }\end{array}$ & oui & $\begin{array}{l}\text { arrive, finti, } \\
\text { sorti }\end{array}$ & $\begin{array}{l}\text { whe fois + } \\
\text { participe }\end{array}$ \\
\hline $\begin{array}{l}\text { participe } \\
\text { présent }\end{array}$ & oui & teart & $e n+$ particjpe \\
\hline
\end{tabular}

Dans la description syntaxique, les données orales constituent des compléments souvent précieux aux exemples écrits (plus fidèles à la norme) ou aux exemples fabriqués (plus sensibles aux buts visés). Il s'agit toutefois de données délicates qui réclament une extrême vigilance (tant dans le choix des situations d'enregistrements que dans la transcription elle-même). Il nous a paru nécessaire d'insister sur les phases antérieures (comme la constitution des données, la transcription et la vérification), souvent passées sous silence, qui apportent pourtant la garantie que les données présentées seront fiables et exploitables'. C'est même une phase essentielle si l'on veut pouvoir ensuite développer une linguistique de l'attesté qui rende compte de l'usage. Il faut pour cela disposer de corpus variés et de grande taille pour faire émerger la répartition des faits de langue en tenant compte de critères externes comme les genres et de phénomènes de lexicalisation qui, comme on l'a esquissé, occupent une part importante. 


\section{BIBLIOGRAPHIE}

BIBER, D. (1988) : Variation across speech and writing, Cambridge, Cambridge University Press. BILGER, M., CAPPEAU, P. (2003) : Les emplois de « contre » dans les corpus de français parlé et de presse écrite, Recherches linguistiques, 26, 91-111.

BILGER, M., CAPPEAU, P. (2004) : L'oral ou la multiplication des styles, Langage et société, 109, 13-30. BLANCHE-BENVENISTE, Cl. (1998) : Une fois dans la grammaire, Travaux de linguistique, 36, 85-101. BLANCHE-BENVEniste, Cl. (2000) : Corpus de français parlé, M. Bilger (éd.), Corpus. Méthodologie et applications linguistiques, Paris, Champion, Perpignan, Presses universitaires de Perpignan. BLANCHE-BENVENISTE, Cl., JEANJEAN, C. (1987) : Le français parlé - Transcription et édition, Paris, Didier Érudition.

BLANCHE-BENVENISTE, Cl. et alii, (1990) : Le français parlé - Études grammaticales, Paris, Éditions du CNRS.

CAPPEAU, P. (2004) : Les formes disjointes des pronoms sujets, Recherches Sur le français parlé, 18, Aix-en Provence, PUP, 107-125.

CAPPEAU, P., DEULOFEU, J. (à paraître) : La quantification des sujets indéfinis à l'oral, Actes du colloque La partition en langue et en discours (Strasbourg).

GADET, F. (1999) : La langue française au Xx siècle - I. L'émergence de l'oral, Chaurand, J. (éd), Nouvelle histoire de la langue française, Paris, Seuil.

HABERT, B. (2000) : Des corpus représentatifs : de quoi, pour quoi et comment ?, M. Bilger (éd.), Corpus. Méthodologie et applications linguistiques, Paris, Champion, Perpignan, Presses universitaires de Perpignan, 11-58.

HALLIDAY, M.A.K. (1985) : Spoken and written language, Oxford, Oxford University Press.

KERBRAT-ORECCHIONI, C., TRAVERSO, V. (2004) : Types d'interactions et genres de l'oral, Langages, 153, 41-51.

MALRIEU, D., RASTIER, F. (2001) : Genres et variations morphosyntaxiques, TAL, 42-2, 547-577, Hermès. MEYER, Ch. F. (2002) : English Corpus Linguistics - An introduction, Cambridge, Cambridge University Press.

MOREAU, M.-L. (1986) : Les séquences préformées : entre les combinaisons libres et les idiomatismes. Le cas de la négation avec ou sans ne, Le Français moderne, 3-4, 137-160.

PUSCH, Cl. D., RAIBLE, W. (éds) (2002) : Romance Corpus linguistics. Corpora and Spoken Language, Tübingen, Gunter Narr Verlag Tübingen.

SABIO, F. (2004) : Les compléments de lieu réalisés par $y$ : description des usages, Recherches Sur le français parlé, 18, 43-62.

VÉRONIS, J. (2004) : Le traitement automatique des corpus oraux, Traitement automatique des langues , 45-2, 1-8. 


\section{NOTES}

1. Certains phénomènes (notamment d'accord) peu représentés dans les corpus rendent toutefois nécessaire l'appui sur des données récupérées à la volée. Car, malgré tous leurs avantages, les corpus disponibles présentent un handicap : leur taille encore trop limitée pour étudier certains faits !

2. Le terme corpus tel qu'il est employé dans ces pages désigne à la fois chaque enregistrement (ou transcription) dans sa singularité et l'ensemble formé par la totalité des enregistrements (ou des transcriptions).

3. Il est peut-être bon de rappeler que parler de gros corpus ne renvoie pas aux mêmes tailles pour l'oral et pour l'écrit. C'est là une constante (quelle que soit la langue) qui tient en grande partie au très lourd travail que représente la mise au propre de corpus oraux (comme la suite de cet article l'illustrera). Si l'on reprend les chiffres publiés dans la Revue de l'Associationfrançaise de linguistique appliquée (RAFLA) de 1997, le déséquilibre est très important entre les 160 millions de mots de Frantext (pour l'écrit) et le million de mots de CorpAix !

4. La tentation exotique reste un danger lorsque l'on travaille sur l'oral. Elle se manifeste à travers les exemples «typés », par exemple ceux qui provoquent le rire, que l'on entend encore quelquefois dans les colloques...

5. On pourra en trouver de nombreux exemples dans la revue Recherches sur le français parlé (Université de Provence). Le site de DELIC fournit la table des matières des numéros parus à l'adresse suivante $:$ http://www.up.univ-mrs.fr/delic/rsfp/index.html.

6. Bilger \& Cappeau (2003).

7. Ce corpus (identifié par FR) présenté dans Recherches sur le français parlé $\mathrm{N}^{\circ} 18$ a été réalisé par l'équipe DELIC et comporte environ 440000 mots. Il a été bâti en tenant compte de critères tels que : l'âge des locuteurs, la région, le niveau scolaire, le caractère préparé/non préparé des échanges, le type professionnel, public ou privé de la parole produite.

8. Pour des exemples plus précis de l'intérêt des genres à l'oral, on renvoie à KerbratOrecchioni \& Traverso (2004) ou à Bilger \& Cappeau (2004).

9. Gadet (1999: 654) s'arrête sur cet emploi du parlé dans l'écrit et précise que y a

l'avantage d'être un mot existant, ce qui explique probablement qu'il soit favorisé dans la graphie du son /i/ (comme dans t'y au lieu t-il).

10. On les trouve notamment dans Blanche-Benveniste et alii (1990). La dernière version est présentée dans RSFP 18, 27-40.

11. L'absence de ponctuation peut dérouter dans un premier temps, mais cette difficulté s'estompe assez vite avec l'habitude.

12. Outre le fait que la taille de cet article ne permettrait pas de répondre à une ambition aussi large (puisqu'on peut penser que ce relevé ne tiendrait pas en quelques pages...).

13. Blanche-Benveniste et alii (1990) ont fait le point de nos connaissances dans différents domaines sans prétendre avoir dressé un bilan complet.

14. Ces quelques observations ne porteront que sur des faits de micro-syntaxe. Pour la macro-syntaxe on renvoie, par exemple, à Blanche-Benveniste et alii (1990).

15. Il s'agit d'un logiciel qui permet de disposer, très rapidement, de la forme recherchée insérée dans une ligne (qui fournit ainsi un contexte droit et un contexte gauche réduits). Cet outil est très utile en syntaxe mais présente un inconvénient : il permet de travailler 
sans prendre nécessairement connaissance du contexte au-delà de la ligne (ce qui peut s'avérer insuffisant) ni du contenu des corpus exploités (ce qui conduit à ne connaître que des portions du corpus).

16. On lira avec profit, sur ce point, l'introduction de Jean Véronis (2004) au volume 45-2 de la revue Traitement automatique des langues portant sur le traitement automatique des corpus oraux.

17. Le TLF ne consacre à cet emploi que quelques lignes en fin d'article.

18. On a utilisé ici un corpus diversifié (de quatre millions de mots) fourni par Jean Véronis (Université de Provence / Delic).

19. Le sujet l'un qui attend la réalisation de l'autre n'est pas visé par cette observation. 20. On peut penser que l'écrit qui a servi de support influence ce type d'emploi. C'est, selon nous, le lien qui autorise le rapprochement entre ces deux participes « directs ». 21. On peut d'ailleurs s'étonner (pour le regretter) que l'édition de corpus ne soit pas valorisée comme une activité scientifique à part entière...

\section{AUTEUR}

PAUL CAPPEAU

Université de Poitiers

Paul.Cappeau@univ-poitiers.fr 